\title{
Parametric Design on Stubble-cutting Disc with Oblique Ripples Based on Reverse Engineering Methods
}

\author{
Ping Zhao, Xuewei Bai, Yongkui $\mathrm{Li}^{*}$, Yue Tian and Changyi Lv \\ Engineering College, Shenyang Agricultural University, Shenyang, 110866, China \\ *Corresponding author
}

\begin{abstract}
Aiming at the design and manufacture difficulty of stubble-cutting disc with oblique ripples, taking one made by America as research object, a kind of computer aided design method was proposed, which was fulfilled in the basis of the structural parameters that were obtained through reverse engineering technology. The point cloud data were obtained by using a Digital Structure Light 3D scanner and a binocular stereoscopic vision system, then the structural equation and parameters was acquired by fitting the data of feature points extracted from the point clouds through boundary detected and curvature calculation. On this basis, 3D parametric model of the stubble-cutting disc with oblique ripples was created in a computer aided design system of Solidworks, which will provide a foundation for its engineering analysis, parameter optimization and mould manufacture. At the same time, this work broadens the range of application of reversing engineering, and also will provide references for researching the application of reverse engineering in the design and manufacture of agricultural machinery.
\end{abstract}

Keywords-computer vision; parametric design; reverse engineering; reverse solution of structural parameter; stubble-cutting disc

\section{INTRODUCTION}

Stubble-cutting sword is the key component of no-tillage seeding, which is required to have better performances of cutting stubble and preventing block. The researches[1-3] shown Stubble-cutting disc with oblique ripples has the advantages of less resistance, strongly block-preventing property, bigger ditching width, smaller disturbance to the soil, etc. But its design and manufacture are extremely difficult in China, nowadays, which is studied through drawing lessons from foreign technology, designers' experience and the tests [2].

Reverse Engineering (RE) is a hot topic in modern design and manufacturing field at present, and it has been widely used in aerospace, aviation, automotive, bio-medicine, archaeological relics copy, bionic engineering, etc. Reverse engineering technology commonly starts with a measurement of the solid model; then reconstructs a geometric model based on coordinate data derived from the measurement system; in the end fulfills rapid prototyping, mold manufacture or redesign. In this process, reconstructing curved surface and 3D model using the coordinate data is one of the important aspects. There are two approaches generally to construct curved surface by point cloud data: (1) constructing curved surface directly by the point cloud, (2) fitting curve by the points, and then constructing the surface by the fitted curve. For a long time, the focus of the RE has been these aspects, occasionally, been used in other respect, such as Kamran Mohaghegh etc [4], proposed a new method of reversing engineering that incorporate construction geometry and design intent. However, as to a part whose shape is complex, frequently curvature variation and relatively regular, the two methods of constructing curved surface by point cloud data will generate bigger error or even impossible. So authors introduce a different viewpoint which focuses on the part's geometry. Namely, a new approach in reverse engineering of such a complex and regular part (Stubble-cutting sword) was proposed, in which a combined reverse engineering technology was used to acquire the part's structural parameters and equations, then to construct 3D model through parameters, which is much more valid.

This study fulfilled reverse solution of parametric equation of the stubble-cutting disc with oblique ripples made in America by using reverse engineering technology, and then the three-dimensional (3D) model was reconstructed according to obtained parameters, which will provide a new method for its design, and pave the way for its redesign and mould manufacture.

\section{II}

Digital MEASUREMENT OF THE STUBbLE-CUTTING DisC WITH OBLIQUE RIPPLE

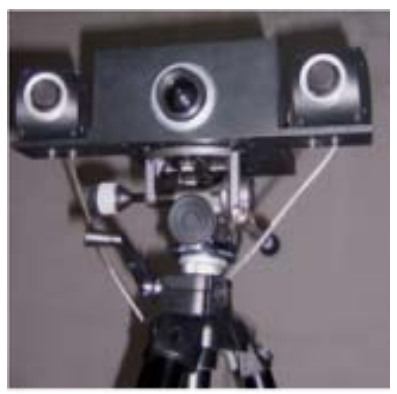

FIGURE I. THE MAIN PART OF 3D SCANNER

In this study, the point cloud data were obtained through a Digital Structure Light (DSL) 3D(three dimensional) scanner based on binocular vision system; it is composed of a computer, 
a projector for projecting a beam of structured light, two CCD cameras, 1394 video capture card, some cables and software system. The main parts of 3D scanner are shown in Figure I, its scan scope and time are $400 \times 300 \sim 200 \times 150 \mathrm{~mm}^{2}$ and $5 \mathrm{~s}$ respectively.

\section{A. The Scheme Design and Planning}

The stubble-cutting disc with oblique ripples is shown in Figure II (a). In order to avoid the influence of surface reflection, the differences of color and curvature, and other factors on image quality, the developer was evenly sprayed on its surface, and the labels were stuck on it before measuring, as shown in Figure II (b). The labels serve two purposes: (1) as identification points while scanning; (2) as datum points while jointing point cloud data obtained through scanning twice.

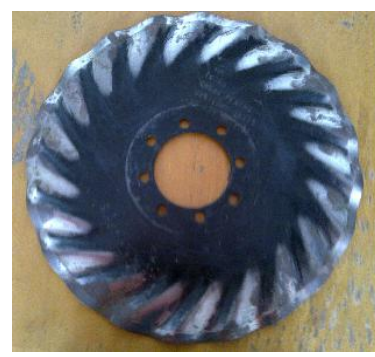

(A)BEFORE PROCESSING

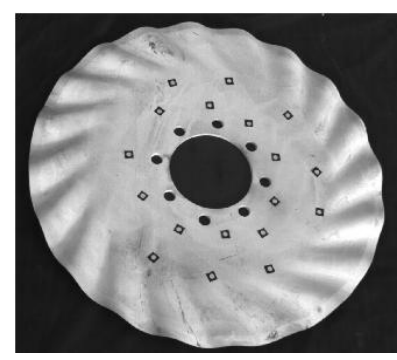

(B) AFTER PROCESSING

\section{FIGURE II. THE STUBBLE-CUTTING DISC WITH OBLIQUE RIPPLES BEFORE AND AFTER PROCESSING}

\section{B. The Measurement of Boundary Curve Points}

Obtaining the boundary points aims at acquiring its structure and parameters. The measuring method based on binocular stereovision was used to obtain $3 \mathrm{D}$ coordinates of boundary curve.

\section{1) Image acquisition}

The left and right images were taken through binocular vision system of 3D scanner that has been calibrated by using the method of reference [5]. At this point, the 3D scanner neither launched grating nor scanned surface.

\section{2) Image processing}

In order to obtain better edge of the stubble-cutting disc with oblique ripples, acquired images were processed in Matlab software by image processing techniques before detecting edge, including binarization, de-noising.

Binarization means that the whole image is only black and white. According to the difference between target and background in gray characteristics, we may regard the image as the combination of target and background with different grayscale. The key is to select optimal threshold. When the gray value of a pixel is greater than the threshold, the pixel longs to interested target, and its color is white. Conversely, the pixel belongs to background, and its color is black. In this study, the optimal threshold is obtained by function graythresh() in MATLAB. It analyzes automatically grayscale histogram, and then determines the optimal threshold according to histogram.
The aim of image de-noising is to reduce random noise that will worsen image quality. There are a lot of de-noising methods that can be divided into two kinds, one kind is the method based on time domain, and another is the method based on frequency domain. The main methods of time-domain de-noising are the weighed smoothing, median filtering, and mathematical morphology. The main methods of frequency-domain de-noising are classic digital filtering, homomorphism filtering and wavelet analysis. In this paper, we adopt median filtering. This method can restrain disturbing pulses and pointlike noise and keep image edge better. Median filtering is finished by function medfilt2() in MATLAB in this study.

\section{3) Edge detection}

At present, the approaches of edge detection mainly are based on differential operation. Differential operation includes first differential calculus and second differential calculus; the edge detection operators based on first differential calculus include Roberts operator[6], Sobel operator, Prewitt operator and Canny operator[7], etc. the edge detection operators based on second differential calculus include Laplacian operator and LOG(Laplacian of Gaussian) operator. A lot of experiments were finished according to all kinds of operators, but, the results weren't favorable. In this study, considering the specific object, we used mathematical morphological method to finish edge detection, which could be finished by function bwmorph(*,'remove') in MATLAB.

4)

\section{Edge corner detection and stereo matching}

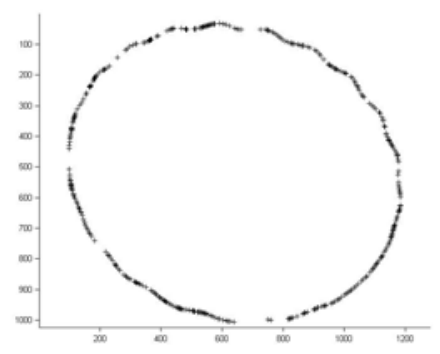

\section{(A) CORNER POINTS IN LEFT IMAGE}

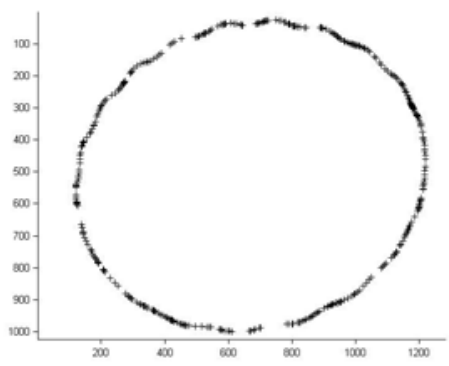

(B) CORNER POINTS IN RIGHT IMAGE

FIGURE III. DETECTED EDGE CORNER POINTS OF THE STUBBLE-CUTTING DISC WITH OBLIQUE RIPPLES

In order to improve matching speed, the corner points of edge in two images were extracted by using Harris operator[8], on this basis, stereo matching was realized with the combination 
of feature matching and area matching under epipolar constraint, namely, take the corner points of edge as matching objects, epipolar constraint[9] and regional correlation were used to finish the edge matching of left and right image. Epipolar constraint can reduces the search space to one dimension (along the epipolar lines)[10]. Regional correlation was resolved by NCC(Normalized Cross Correlation) [11]. According to proposed method, extracted corner points are shown in Figure III.

\section{5) 3D coordinates calculation in binocular vision}

In binocular stereo vision, $\left(X_{c L}, Y_{c L}, Z_{c L}\right)$ and $\left(X_{c R}, Y_{c R}, Z_{c R}\right)$ are the coordinates of a given point in space in left and right camera coordinate system respectively; $\left(x_{L}, y_{L}\right)$ and $\left(x_{R}, y_{R}\right)$ are the coordinates of left and right image points in left and right image coordinate system respectively. The effective focal lengths of left and right camera are $f_{L}$ and $f_{R}$ respectively. The equations as follows are obtained according to pinhole imaging and perspective transformation model.

$$
\begin{gathered}
Z_{c L}\left[\begin{array}{l}
x_{L} \\
y_{L} \\
1
\end{array}\right]=\left[\begin{array}{ccc}
f_{L} & 0 & 0 \\
0 & f_{L} & 0 \\
0 & 0 & 1
\end{array}\right]\left[\begin{array}{l}
X_{c L} \\
Y_{c L} \\
Z_{c L}
\end{array}\right] \\
Z_{c R}\left[\begin{array}{l}
x_{R} \\
y_{R} \\
1
\end{array}\right]=\left[\begin{array}{ccc}
f_{R} & 0 & 0 \\
0 & f_{R} & 0 \\
0 & 0 & 1
\end{array}\right]\left[\begin{array}{l}
X_{c R} \\
Y_{c R} \\
Z_{c R}
\end{array}\right]
\end{gathered}
$$

The relative positions between left and right cameras can be expressed by rotation matrix $R_{c}$ and translation vectors $T_{c}$ as follows.

$$
\left[\begin{array}{c}
X_{c R} \\
Y_{c R} \\
Z_{c R}
\end{array}\right]=\left[\begin{array}{ll}
R_{c} & T_{c}
\end{array}\right]\left[\begin{array}{c}
X_{c L} \\
Y_{c L} \\
Z_{c L} \\
1
\end{array}\right]
$$

The follow Eq.4 can be obtained by Eq. $1 \sim$ Eq.3.

$$
Z_{c R}\left[\begin{array}{l}
x_{R} \\
y_{R} \\
1
\end{array}\right]=\left[\begin{array}{ccc}
f_{R} & 0 & 0 \\
0 & f_{R} & 0 \\
0 & 0 & 1
\end{array}\right]\left[\begin{array}{ll}
R_{c} & T_{c}
\end{array}\right]\left[\begin{array}{c}
\frac{x_{L}}{f_{L}} Z_{c L} \\
\frac{y_{L}}{f_{L}} Z_{c L} \\
Z_{c L} \\
1
\end{array}\right]
$$

According to Eq.1, Eq.2 and Eq.4, the 3D coordinates $\left(X_{c L}, Y_{c L}, Z_{c L}\right)$ and $\left(X_{c R}, Y_{c R}, Z_{c R}\right)$ in left and right cameras coordinate systems can be obtained when $\left(x_{L}, y_{L}\right),\left(x_{R}, y_{R}\right)$, $f_{L}, f_{R}, R_{c}$ and $T_{c}$ are known. Then, calculating the $3 \mathrm{D}$ coordinates in world coordinate system using the left and right camera external parameters, namely, the relative positions parameters between cameras and world coordinates, which could be obtained by camera calibration.

The edge points of the stubble-cutting disc with oblique ripples were obtained according to matching results and the principle of binocular vision, as shown in Figure IV. We can see that the edge points are smooth as a whole.

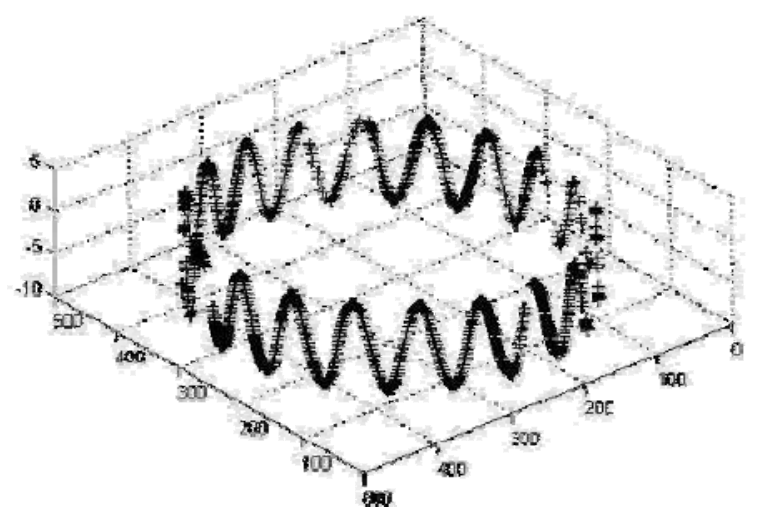

FIGURE IV. ACHIEVED 3D COORDINATES OF THE BOUNDARY CURVE OF THE STUBBLE-CUTTING DISC WITH OBLIQUE RIPPLES

\section{The Measurement of 3D Data Points in Inner Part}

The size of stubble-cutting is beyond single measurement scope of the 3D scanner, so, the whole surface data were obtained through the method that two-time measurement data were automatic registered to combine a uniform model. The point clouds are shown in Figure V.

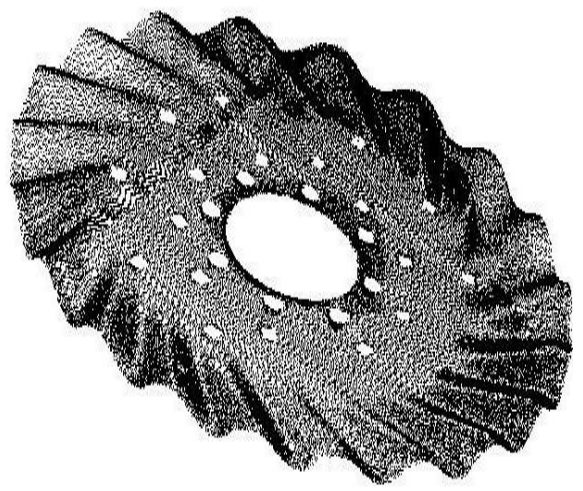

FIGURE V. SURFACE POINT DATA OF THE STUBBLE-CUTTING DISC WITH OBLIQUE RIPPLES 
III

\section{REVERSE SOLUTIONS OF STRUCTURAL PARAMETERS AND EQUATIONS}

\section{A. Structural Parameters of Stubble-cutting Disc with Oblique Ripples}

The gauffers of disk sword with ripples are defined as sword ridge. The sword whose ridge lines pass through the center is defined as centripetal stubble-cutting disc, whereas the sword whose ridge lines don't pass through the center is defined as oblique stubble-cutting disc. As shown in Figure VI, OC is the ridge line of centripetal stubble-cutting disc; $\mathrm{BC}$ is the ridge line of stubble-cutting disc with oblique ripples. The main structural parameters are outer radius of ripples $(\mathrm{R})$, inner radius of ripples $(\mathrm{r})$, ripple angle $(\alpha)$, the number of ripples $(\mathrm{Z})$, the thickness of sword (h), the curve of outer profile and its amplitudes (A).

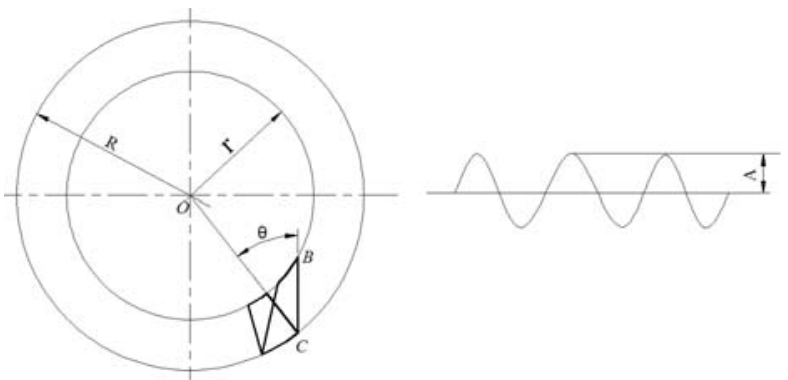

FIGURE VI. STRUCTURAL PARAMETERS OF THE STUBBLE-CUTTING DISC WITH OBLIQUE RIPPLES

\section{B. Reverse Solutions of Structural Parameters and Equations}

1) The curve equation of outer profile and outer radius of the ripples

The curve equation of outer profile and outer radius of the ripples were acquired through the boundary data of outer profile [Figure IV], the methods and steps are as follows:

(1) Obtain the outer radius and center through fitting into a circle using $\mathrm{x}$ and $\mathrm{y}$ coordinates of boundary points; $\mathrm{R}$ was $213.87 \mathrm{~mm}$, and center coordinates were $(212.58,283.06)$;

(2) Make the center of fitted circle coincide with the origin of coordinate system by using coordinate translation;

(3) Convert the $3 \mathrm{D}$ coordinate $\operatorname{system}(\mathrm{x}, \mathrm{y}, \mathrm{z})$ into cylinder coordinate system $(r, \theta, z)$, and solve the corresponding $\theta$ values of boundary data;

(4) Solve the functional relation between $\mathrm{z}$ and $\theta$ through data fitting method. The sine curve fitting algorithm was selected after contrasts of several fitting methods. Given sine function: $z=A \sin (b \theta+c)$, the fitting results with a $98 \%$ confidence are shown in Table I.
TABLE I. DATA FITTING RESULTS OBTAINED BY SINE CURVES

\begin{tabular}{|l|l|l|l|l|}
\hline $\begin{array}{l}\text { Fitting } \\
\text { parameters }\end{array}$ & $\begin{array}{l}\text { Fitting } \\
\text { results }\end{array}$ & $\begin{array}{l}\text { Confidenc } \\
\text { e interval }\end{array}$ & $\begin{array}{l}\text { Root mean } \\
\text { square } \\
\text { error }\end{array}$ & $\begin{array}{l}\text { Correlative } \\
\text { coefficient }\end{array}$ \\
\hline$A$ & 5.082 & $\begin{array}{l}5.014-5.13 \\
1\end{array}$ & & \\
\cline { 1 - 3 } b & 19.26 & $\begin{array}{l}19.24-19.2 \\
8\end{array}$ & \multirow{2}{*}{0.5082} & 0.9708 \\
\cline { 1 - 2 } & 2.15 & $\begin{array}{l}2.103-2.19 \\
6\end{array}$ & & \\
\hline
\end{tabular}

The contrast relation between measured data points and fitting curve is shown in Figure VII.The fitting results indicate the profile curve of ripples really is a sine curve. Outer profile curve of ripples in 3D coordinate system, which was obtained by its structural parameters, is shown in Figure VIII.

2) Reverse solution of inner radius of ripples $(r)$ and ripple angle $(\alpha)$

Equations The position at inner radius of ripples is a transition from curved surface to plane, so its curvature is strongly variational, which is the same pattern as the position of ridge line. Those make curvature calculation available to extract the data of inner radius and ridge line.

Inner boundary data of ripples was extracted through only single scanning data in order to avoid errors caused by data integrating. The single scanning data is shown in Figure IX, and obtained points of ripples' profile through curvature calculation are shown in Figure X, in which the "+" are the inner boundary points of ripples.

The inner boundary points were fitted into a circle, with the radius being $149.16 \mathrm{~mm}$, namely, $\mathrm{r}=149.16 \mathrm{~mm}$.

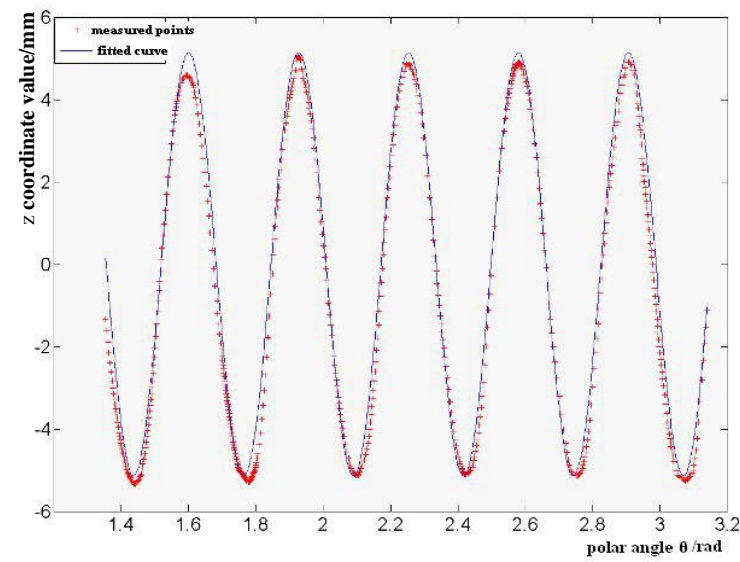

FIGURE VII. COMPARISON DIAGRAM BETWEEN MEASURED BOUNDARY DATA POINTS AND THEIR FITTING CURVE 


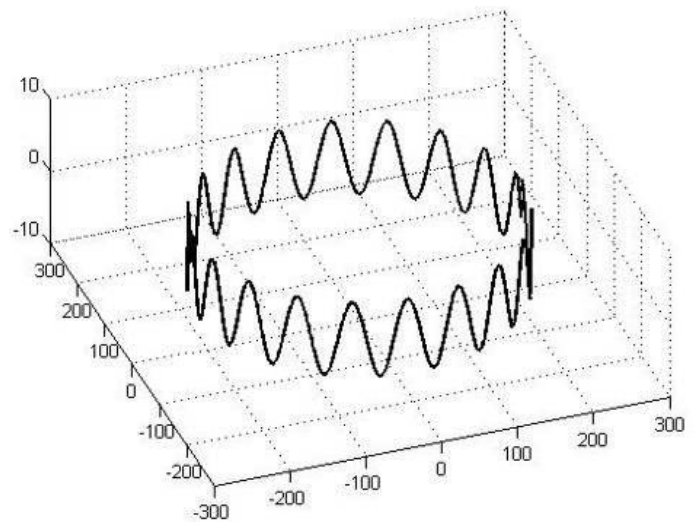

FIGURE VIII. OUTER PROFILE CURVE OF RIPPLES OBTAINED BY ITS STRUCTURAL PARAMETERS IN 3D COORDINATE SYSTEM

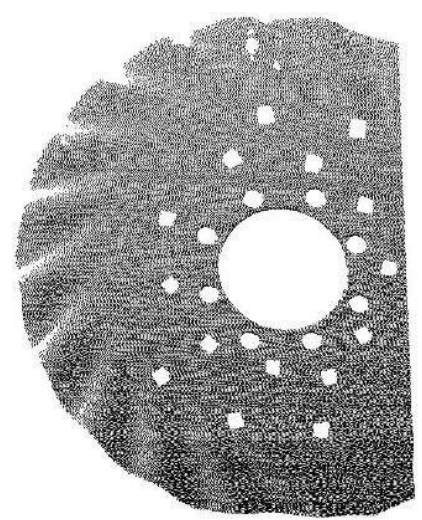

FIGURE IX. SINGLE SCANNING DATA

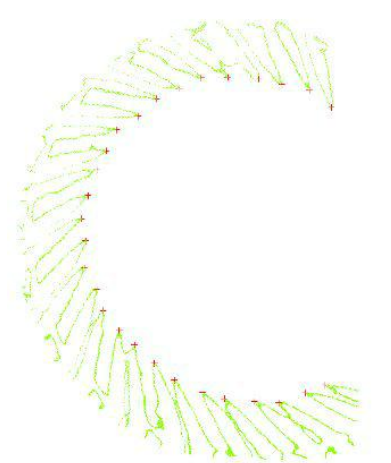

FIGURE X. EXTRACTED INNER BOUNDARY POINTS OF RIPPLES

The reverse solution of ripple angle $(\alpha)$ was obtained by extracting sword ridge line that could be got from the curvature calculation result of ripple profile based on color feature. As shown in Figure XI, the continuous line is ridge line acquired through a ripple profile. One ridge line could be obtained a ripple angle, the final ripple angle $(\alpha)$ was the average value of those, and it was $29.702^{\circ}$.

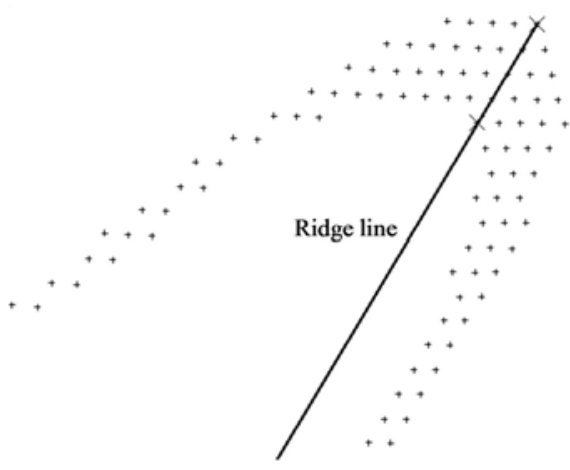

FIGURE XI. THE PROFILE POINTS OF A RIPPLE AND OBTAINED RIDGE LINE

\section{The CREATING OF 3D MOdel}

The 3D model was created through using obtained the structural parameters in a computer aided design system of Solidworks, and the results are shown in Figure XII, which will provide the basis for subsequent engineering analysis, parameter optimization and mould manufacture. Compared with 3D model reconstruction based on point cloud data, the advantage of model reconstruction based on structural parameters is that it fulfills the parametric design, and in this instance the model structure can vary with its parameters, fulfills authentic parametric design.
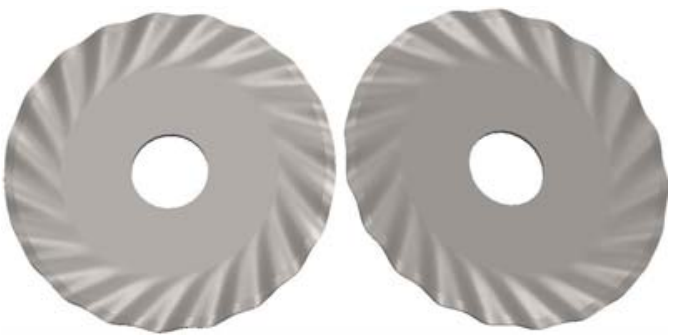

FIGURE XII. 3D MODEL OF STUBBLE-CUTTING DISC CREATED THROUGH OBTAINED STRUCTURAL PARAMETERS

\section{CONCLUSIONS AND RESUlts}

(1) The reverse solution of structural parameters and equation of stubble-cutting disc with oblique ripples were well fulfilled through reverse engineering technology. The results show that the outer profile of ripples is sine curve, as for the given stubble-cutting disc with oblique ripples made by America, the equation is $z \quad 5.082 \sin (19.26 \quad 2.15)$, and its ripple angle $(\alpha)$ is 29.702 , outer radius of ripples $(\mathrm{R})$ is $213.87 \mathrm{~mm}$, inner radius of ripples(r) is $149.16 \mathrm{~mm}$.

(2) The reconstruction of the $3 \mathrm{D}$ parametric model of stubble-cutting disc with oblique ripples provides the foundation for its parameter optimization and mould manufacture, and the research results provide a new method for its design and manufacture. At the same time, this research also provides a reference for the application of reverse engineering technology in the design and manufacture of agricultural machine.

(3) The research results also illustrate that RE can be used to obtain the parameters and equations of the regular structures. 


\section{ACKNOWLEDGEMENT}

The digital measurement method in this research was supported by the National Science Foundation of China (51505305) and Doctoral Fund of Ministry of Education of China (20132103120016).

\section{REFERENCES}

[1] Zhao Xu, Zhang Zuli, Tang Ping, Zhang Guoliang, Zhang Weizheng, Behavior of Passive Stubble-cutting Disc with Oblique Ripples, Transactions of the Chinese Society for Agricultural Machinery, 42(2011)64-67.

[2] Zhang Zuli, Zhao Xu, Bai Xiaohu, Zhang Xudong, Kinematics Analysis and Simulation of Disc Coulter with Oblique Ripple, JOURNAL OF AGRICULTURAL MECHANIZATION RESEARCH, 31(2009) 27 -30.

[3] Zhao Xu, Zhang Zuli, Bai Hongchun, Zhang Guoliang, Zhang Xudong, The New Type Residues Cutting and Anti-blocking Device, JOURNAL OF AGRICULTURAL MECHANIZATION RESEARCH, 31(2009) 58-61.

[4] Kamran Mohaghegh, Mohammad H. Sadeghi, Amir Abdullah, Reza, Improvement of reverse-engineered turbine blades using construction geometry, The International Journal of Advanced Manufacturing Technology, 49(2010) 675-687.

[5] Ping Zhao, Yong-kui Li, Li-jun Chen, Xue-wei Bai, Camera calibration technology based on circular points for binocular stereovision system, Communications in Computer and Information Science,158(2011) 356-363.

[6] Roberts L G, Machine Perception of Three-Dimensional Solids, In Proceedings of Optical and Electro-optical Information Processing, 1965, pp.159-197.

[7] JOHN CANNY, A Computational Approach to Edge Detection, IEEE Transactions on Pattern Analysis and Machine Intelligence, 1986, pp.679-698.

[8] Chris Harris, Mike Stephens, A COMBINED CORNER AND EDGE DETECTOR, In Proceedings of the 4th Alvey vision conference, 1988, pp.189-192.

[9] Larry S.Shapiro, Andrew Zisserman, Michael Brady, Motion From Point Matches Using Affine Epipolar Geometry, Lecture Notes in Computer Science, 801(1994)73-84.

[10] Ingemar J.Cox, Sunita L.Hingorani, Satish B.Rao, A Maximum Likelihood Stereo Algorithm, Computer Vision and Image Understanding, 63(1996)542-567.

[11] Kai Briechle, Uwe D.Hanebeck, Template Matching Using Fast Normalized Cross Correlation, In Proceedings of SPIE, V.4387, Optical Pattern Recognition XII, Orlando, FL. 2001, pp.95-102. 